\title{
202. The Existence of Partial Mirrors in Wollastonite
}

\author{
By Tei-ichi Ito, M. J. A., Ryoichi SAdanaga, Yoshio Takéuchi, \\ and Masayasu ToKoNAMI*) \\ Mineralogical Institute, Faculty of Science, University of Tokyo
}

(Comm. Dec. 12, 1969)

It has been pointed out (Ito, 1950) that the structures of wollastonite $\left(\mathrm{CaSiO}_{3}\right)$ and parawollastonite (ibid.) exhibit 'extra' reflection-symmetry beside the symmetry characteristic of the respective space group. This reflection plane is different from one constituting the space group in that it is discontinued on both sides as shown in Fig. 1, and is consequently effective only within a limited but definite portion of the unit cell. Symmetry operations of this kind were later named by Dornberger-Schiff (1956) 'partial' symmetry operations. Accordingly we now call them simply partial mirrors. As demonstrated already, the mutual staggering between the partial mirrors is just a quarter of a unit length, in this case the $b$-length. From these findings, it has been concluded that the polymorphic substance may be composed of the same common units, and that different modes of twinning (rather than different unit cells) will produce different structures, for example, gliding $G^{1}$ (echelon) and gliding

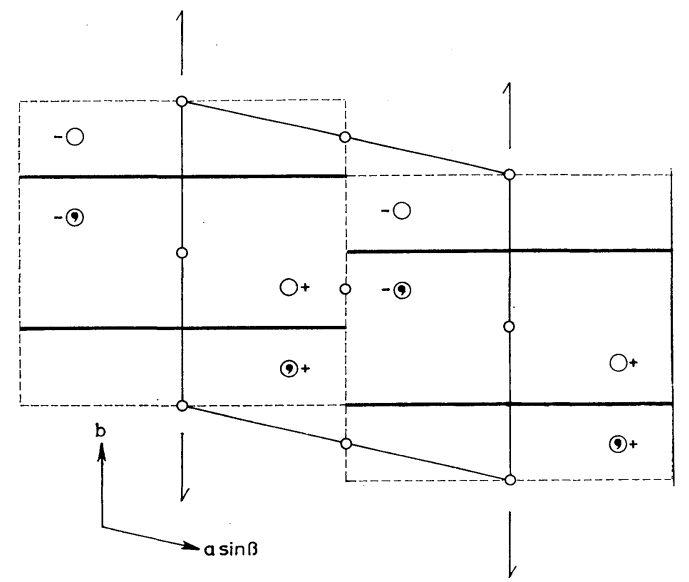

Fig. 1. The unit cell and symmetry of wollastonite. Projection along the $c$-axis. Thick lines represent partial mirrors perpendicular to the $b$-axis, and broken lines the protowollastonite units in wollastonite.

*) Present address: Institute of Scientific and Industrial Research, Osaka University, Senri, Osaka. 
$G^{2}$ (alternate), giving rise to wollastonite on one hand and parawollastonite on the other (Ito, 1950, p. 4 and 109).

The presence of the polysynthetic structures in general and that of partial mirrors in particular seem to have opened up a new vista on the nature of crystal. Crystals consist, indeed, of atoms or molecules. They may, however, be looked upon as having a building unit other than atoms or molecules, the unit being not only, as usual, the unit cell of the respective crystal but also its multiple or submultiple. Further, the units are stacked together in parallel and continuous positions for the most part. In certain cases, however, they are arranged in staggering and discontinuous positions.

Though a visual inspection sufficed to convince us of the presence of partial mirrors in wollastonite, we have undertaken a refinement of its crystal structure in order to ascertain to what extent of exactitude they are realized in it.

\section{Refinement and description of the structure.}

The specimen of (triclinic) wollastonite employed in the present experiment was from Hokizawa, Japan (Ito, 1935), and is the same as used in the previous study (Ito, 1950, p. 93). Intensity data about $1,500 \mathrm{hkl}$ reflections were collected from its Weissenberg photographs $(\mathrm{CuK} \alpha)$. Intensity statistics by the Wilson method was applied to the data and the space group of the crystal was confirmed to be $P \overline{1}$. For the cell dimensions, the current values : $a=7.94 \AA, b=7.32 \AA$, $c=7.07 \AA, \alpha=90^{\circ} 02^{\prime}, \beta=95^{\circ} 22^{\prime}, \gamma=103^{\circ} 26^{\prime}$, were considered to be sufficiently accurate for the present purpose.

The processes of refinement were initiated with the coordinates of atoms given by Mamedov and Belov (1956), and after several repetitions of least square processes, the final coordinates as listed in Table I were deduced. The $R$-factor, 0.36 for the starting structure, was reduced to 0.21 , and when unobserved reflections were given the value $\left|F_{\min } / \sqrt{3}\right|$, the factor became to be 0.19 . The three-dimensional Fourier analysis was carried out, and the accuracy of the structure determination was then assessed with the aid of the Cruickshank method (Cruickshank, 1949), and the standard deviations for $\mathrm{Ca}, \mathrm{Si}$ and $\mathrm{O}$ atoms were estimated to be $0.001,0.0014$ and 0.004 in decimal coordinates respectively.

The structure is shown projected in the $c$-direction in Fig. 2. As will be recognized from it, the structure may be regarded as built up of $\mathrm{SiO}_{3}$ chains and $\mathrm{Ca}_{3} \mathrm{O}_{8}$ laths, both running parallel to the $b$-axis, sharing oxygen atoms among them, as has been already described by Liebau (1957).

Judging from the distribution of bonds, however, we may also conceive of the structure as composed of the layers parallel to (100), 
Table I. Coordinates of atoms in wollastonite

\begin{tabular}{l|c|c|c}
\hline & $x$ & $y$ & $z$ \\
\hline Cal & -.002 & $.249_{5}$ & -.026 \\
Call & .700 & -.075 & .264 \\
CaII' & .698 & $.424_{5}$ & .261 \\
SiI & .314 & .043 & .229 \\
SiI & .314 & .614 & .229 \\
SiII & .102 & $.275_{5}$ & .442 \\
OI & .100 & .275 & .666 \\
OII & .076 & .769 & .693 \\
OIII & .194 & .062 & .037 \\
OIII' & .194 & .535 & .037 \\
OIV & .516 & .129 & .226 \\
OIV & .520 & .630 & .233 \\
OV & .224 & .120 & .405 \\
OV' & .224 & .492 & .405 \\
OVI $^{\prime}$ & .290 & $.822_{5}$ & .260 \\
\hline
\end{tabular}

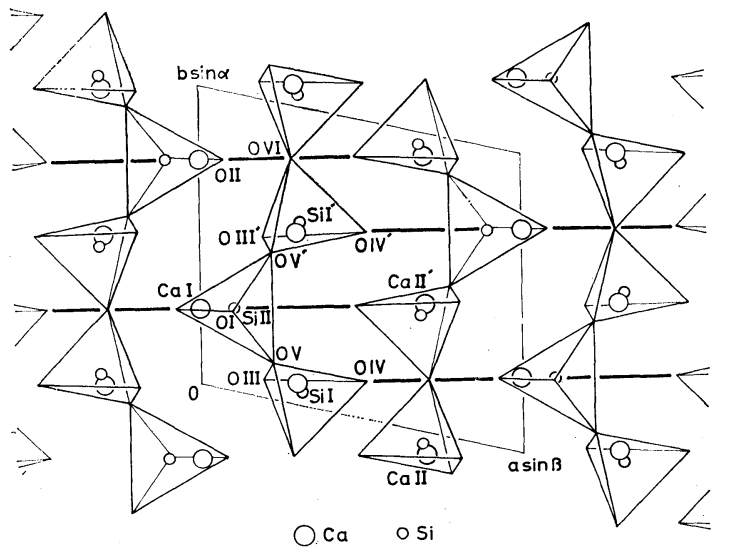

Fig. 2. Structure of wollastonite projected along the $c$-axis. Oxygen atoms are at the corners of the tetrahedra. Thick lines represent partial mirrors.

the bonds linking these layers together being much weaker than those within a layer.

2. Partial mirrors in the refined structure.

In order to facilitate the comparison of wollastonite and protowollastonite units within it, a transformation of axes of the former was introduced (Fig. 3), obtaining the modified sets of coordinates as listed in Table II. Then if the protowollastonite unit incorporated in the wollastonite cell is exactly reflection-symmetric, as defined above, atoms on the partial mirrors should have 0.250 or 0.750 as 


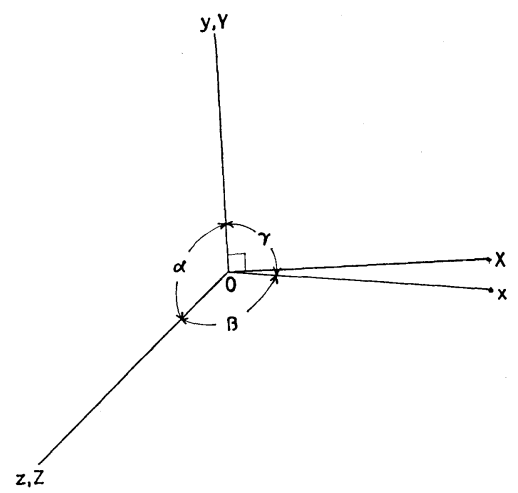

Fig. 3. Axial transformation for examination of the partial mirror symmetry of wollastonite. Capital letters express the new axes.

Table II. Coordinates of atoms after axial transformation

\begin{tabular}{|c|c|c|c|}
\hline & $X$ & $Y$ & $Z$ \\
\hline $\mathrm{CaI}$ & -.002 & $.250=.250+.000$ & -.026 \\
\hline $\mathrm{CaII}$ & -.300 & $-.005=.250-.255$ & .264 \\
\hline * $\left\{\mathrm{CaII}^{\prime}\right.$ & -.302 & $.495=.250+.245$ & .261 \\
\hline SiI & .314 & $-.030=.250-.280$ & .229 \\
\hline$*\left\{\mathrm{SiI}^{\prime}\right.$ & .314 & $.541=.250+.291$ & .229 \\
\hline SiII & .102 & $.252=.250+.002$ & .442 \\
\hline $\mathrm{OI}$ & .100 & $.252=.250+.002$ & .666 \\
\hline OII & .076 & $.751=.750+.001$ & .693 \\
\hline (OIII & .194 & $.017=.250-.233$ & .037 \\
\hline$*\left\{\mathrm{OIII}^{\prime}\right.$ & .194 & $.490=.250+.240$ & .037 \\
\hline $\int \mathrm{OIV}$ & .516 & $.009=.250-.241$ & .226 \\
\hline$*\left\{\right.$ OIV $^{\prime}$ & .520 & $.509=.250+.259$ & .233 \\
\hline $\mathrm{OOV}$ & .224 & $.068=.250-.182$ & .405 \\
\hline$*\left\{V^{\prime}\right.$ & .224 & $.440=.250+.190$ & .405 \\
\hline OVI & .290 & $.755=.750+.005$ & .260 \\
\hline
\end{tabular}

*) Pair of atoms supposed to be located symmetrically above (primed) and below a partial mirror.

their $Y$-coordinates, and each of those located apart from a mirror should have its counterpart with the same $(X, Z)$ coordinates and the same length of separation from the mirror but in the opposite direction. In fact, a glance at Table II will reveal at once that CaI, SiII, OI, OII and OVI are atoms of the former category, and the pairs, (CaII, $\left.\mathrm{CaII}^{\prime}\right)$, ( $\left.\mathrm{SiI}, \mathrm{SiI}^{\prime}\right)$, (OIII, OIII'), (OIV, OIV') and $\left(\mathrm{OV}, \mathrm{OV}^{\prime}\right)$ fall into the latter.

The deviations of some of them from those of the ideal configuration, especially concerning $Y$-coordinates as given in Table II, exceed 
in certain amounts the standard deviations estimated earlier. At this stage of analysis, the structure of wollastonite we have arrived at, therefore, appears to possess the partial mirrors only approximately.

\section{Discussion.}

Firstly we took into account the $Y$-coordinates given in Table II. We notice immediately the deviations of $Y$-coordinates systematically become larger with the increase of the respective $X$-coordinates, and for those expected to be related to each other by a partial mirror, the deviation for the upper atoms (with a prime in Table II) is always larger than that for its lower counterpart. These suggest that if the structure were deformed so as to widen the interfacial angle between (100) and (010) around the $c$-axis passing the origin (vide Fig. 2), all the deviations could be eliminated. In fact, the value $104^{\circ} 29^{\prime}$ instead of the actual $103^{\circ} 24^{\prime}$ is taken for the angle, the deviations of the $Y$-coordinates from those of the ideal structure vanish entirely as seen in Table III. On the other hand, if we extend on both sides the partial mirror in the wollastonite lattice, we find that it does not exactly pass through the lattice points as illustrated in Fig. 4. This means that the mirrors are located in the position irrational to the lattice. Now if the interfacialangle is widen as above, it will assure the lattice and mirror to keep a rational relation. ${ }^{\dagger}$ Indeed, according

Table III. Coordinates of atoms in the "deformed" structure

\begin{tabular}{|c|c|c|c|}
\hline & $X$ & $Y$ & $Z$ \\
\hline $\mathrm{CaI}$ & -.002 & $.250=.250+.000$ & -.026 \\
\hline CaII & -.300 & $.000=.250-.250$ & .264 \\
\hline${ }^{*}\left\{\mathrm{CaII}^{\prime}\right.$ & -.302 & $.500=.250+.250$ & .261 \\
\hline SiI & .314 & $-.035=.250-.285$ & .229 \\
\hline$*\left\{\mathrm{SiI}^{\prime}\right.$ & .314 & $.536=.250+.286$ & .229 \\
\hline SiII & .102 & $.250=.250+.000$ & .442 \\
\hline OI & .100 & $.250=.250+.000$ & .666 \\
\hline OII & .076 & $.750=.750+.000$ & .693 \\
\hline OIII & .194 & $.014=.250-.236$ & .037 \\
\hline$*\left\{\begin{array}{l}\mathrm{OIII}^{\prime} \\
\end{array}\right.$ & .194 & $.487=.250+.237$ & .037 \\
\hline (OIV & .516 & $.000=.250-.250$ & .226 \\
\hline$*\left\{\right.$ OIV $^{\prime}$ & .520 & $.500=.250+.250$ & .233 \\
\hline $\mathrm{OV}$ & .224 & $.064=.250-.186$ & .405 \\
\hline$*\left\{\mathrm{OV}^{\prime}\right.$ & .224 & $.436=.250+.186$ & .405 \\
\hline OVI & .290 & $.750=.750-.000$ & .260 \\
\hline
\end{tabular}

*) Pair of atoms located symmetrically above (primed) and below a partial mirror.

t) Because the alleged deviation of the $\alpha$-angle from $90^{\circ}$ is as small as $2^{\prime}$ and well within the experimental error, it has been neglected in the present consideration. 


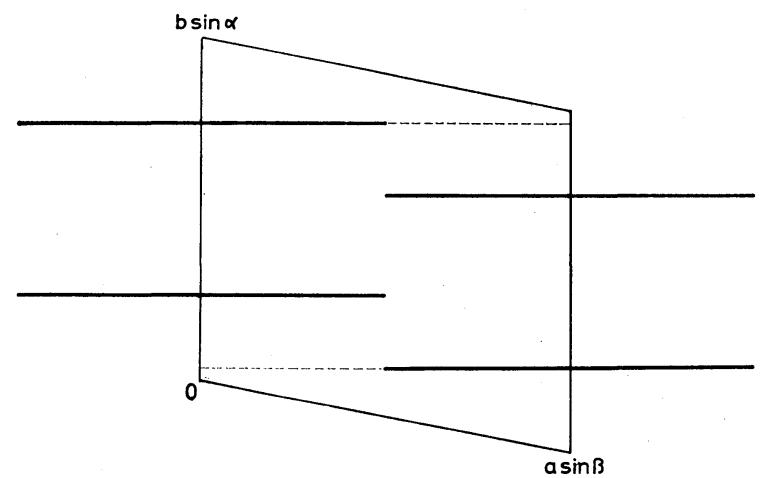

Fig. 4. Irrational relation between the crystal lattice and the partial mirrors in wollastonite. Thick lines represent partial mirrors, and the misfit is shown somewhat exaggerated.

to Friedel-principle, generalized by Ito,*) there should be no obliquity between the individual and twinned lattices.

Now, if the structure is strictly reflection-symmetric across the partial mirrors, a pair of atoms related to each other by a mirror will have the same $(X, Z)$ coordinates. Inspection of Table III informs us that this holds good except for the pairs (CaII, CaII') and (OIV, OIV'), for each of these pairs, the difference in $Z$-coordinates exceeding somewhat the standard deviation of the respective atom. These atoms are situated in the structure on the outermost sides of the layer binding layer to layer and should not be subjected to such a simple deformation procedure as we have adopted to simulate the actual structure to the ideal one.

In conclusion, although the final structure is not completely identical with the ideal structure with partial mirrors, the similarity between them is deemed enough to warrant us to support the existence of partial mirrors in wollastonite. A detailed report of our study together with experimental data obtained will be published elsewhere shortly.

\section{References}

Cruickshank, D. W. J. (1949): Acta Cryst., 2, 65.

Dornberger-Schiff, K. (1956): Acta Cryst., 9, 593.

Friedel, G. (1926) : Leçons de Cristallographie. Berger-Levrault, Paris.

Ito, T. (1935) : Beiträge zur Mineralogie von Japan. Neue Folge, I, 183.

- (1950): X-Ray Studies on Polymorphism. Maruzen, Tokyo.

Liebau, F. (1957): Naturwis., 44, 178.

Mamedov, K. H., and Belov, N. V. (1956): Compt. Rend. Acad. Sci., USSR, 107, 463.

*) Friedel (1926) advocated the ease of twin formation when no obliquity is met with, and Ito (1950) pointed out that non-obliquity is one of the conditions essential to the formation of the polysynthetic structure. 